\title{
Adesão de pessoas com diabetes mellitus tipo 2 ao tratamento medicamentoso
}

\author{
Adherence of people with type 2 diabetes mellitus to drug treatment \\ Adhesión de las personas con diabetes mellitus tipo 2 al tratamiento medicamentoso
}

\author{
Lilian Cristiane Gomes-Villas Boas', Maria Cristina Foss-Freitas", Ana Emilia Pace ${ }^{\prime \prime \prime}$ \\ ' Universidade de São Paulo, Escola de Enfermagem de Ribeirão Preto, \\ Programa de Pós-Graduação de Enfermagem Fundamental. Ribeirão Preto-SP, Brasil. \\ "Universidade de São Paulo, Faculdade de Medicina de Ribeirão Preto. Ribeirão Preto-SP, Brasil. \\ II' Universidade de São Paulo, Escola de Enfermagem de Ribeirão Preto, \\ Departamento de Enfermagem Geral e Especializada. Ribeirão Preto-SP, Brasil.
}

\section{Submissão: 31-05-2012 Aprovação: 18-01-2014}

\section{RESUMO}

Estudo seccional de abordagem quantitativa que objetivou avaliar a adesão ao tratamento medicamentoso entre pessoas com diabetes mellitus, e sua relação com variáveis clínicas, de tratamento e controle metabólico. A amostra constituiu-se de 162 pessoas com diabetes mellitus tipo 2 em seguimento ambulatorial. Para a coleta dos dados, foram utilizadas a Medida de Adesão aos Tratamentos e a consulta aos prontuários dos participantes. Obteve-se elevada adesão ao tratamento medicamentoso. Para um $\mathrm{p}<0,05$, obteve-se correlação inversa com a pressão arterial diastólica e correlação direta com a frequência diária de administração de insulina e de antidiabéticos orais. Não houve correlações estatisticamente significantes entre adesão e variáveis de controle metabólico. Os resultados divergem da literatura no que se refere à taxa de adesão ao tratamento medicamentoso em doenças crônicas, bem como na correlação entre adesão e complexidade do regime medicamentoso, o que aponta para a necessidade de mais estudos sobre essa temática.

Descritores: Diabetes Mellitus; Cooperação do Paciente; Cuidados de Enfermagem.

\section{ABSTRACT}

This cross-sectional and quantitative study aimed to evaluate the adherence to drug treatment of the people with diabetes mellitus and its relation to clinical, treatment and metabolic control variables. Sample consisted of 162 people with type 2 diabetes mellitus on follow-up outpatient care. The Measure of Treatment Adherence and consultation to the participants' medical records were used for data collection. A high adherence to drug treatment was obtained. For a $p<0.05$, it was obtained an inverse correlation with diastolic blood pressure and a direct correlation with the frequency of daily administration of insulin and oral antidiabetic agents. There were no statistically significant correlations between adherence and metabolic control variables. Results diverge from the literature regarding the adherence to drug treatment in chronic diseases, as well as in the correlation between adherence and complexity of drug regimen, which points to the need for more studies on this theme.

Key words: Diabetes Mellitus; Patient Compliance; Nursing Care.

\section{RESUMEN}

Estudio transversal de enfoque cuantitativo que objetivó evaluar la adhesión al tratamiento medicamentoso de las personas con diabetes mellitus y su relación con variables clínicas, tratamiento y control metabólico. La muestra consistió en 162 personas con diabetes mellitus tipo 2 en seguimiento ambulatorio. Para recolección de datos fueron utilizadas la Medida de Adhesión a Tratamientos y consulta a los registros médicos de los participantes. Se obtuvo una alta adhesión al tratamiento medicamentoso. Para uno $\mathrm{p}<0,05$, se obtuvo una correlación inversa con la presión arterial diastólica y una correlación directa con la frecuencia diaria de administración de insulina y antidiabéticos orales. No hubo correlación estadísticamente significativa entre adherencia y variables de control metabólico. Resultados difieren de la literatura en relación a la tasa de adhesión al tratamiento medicamentoso de enfermedades crónicas, así como la correlación entre adhesión y complejidad del régimen medicamentoso, lo que apunta a necesidad de más estudios sobre este tema.

Palabras clave: Diabetes Mellitus; Cooperación del Paciente; Cuidados de Enfermería.

\section{AUTOR CORRESPNDENTE Ana Emilia Pace E-mail: aepace@eerp.usp.br}




\section{INTRODUÇÃO}

A adesão ao tratamento tem como definição clássica a extensão na qual o comportamento da pessoa coincide com a orientação médica no que se refere, por exemplo, ao uso da medicação, ao seguimento de dietas, a mudanças no estilo de vida ou à adoção de comportamentos protetores de saúde ${ }^{(1)}$.

Referente ao tratamento medicamentoso, a porcentagem de pessoas em falência para usar a medicação conforme a prescrição médica varia de $7 \%$ a $64 \%{ }^{(2-3)}$. Pessoas que sofrem de doenças crônicas com pouco ou nenhum sintoma são mais propensas a não adesão, assim como a ausência de queixas físicas pode representar falta de motivação para o uso das medicações, o que não acontece com as doenças agudas e sintomáticas ${ }^{(4)}$. Entre as pessoas com Diabetes Mellitus (DM), especialmente com o tipo 2 da doença, a adesão ao tratamento medicamentoso tende a ser baixa devido ao seu caráter assintomático, levando à crença de que a medicação não é necessária ${ }^{(5)}$.

A literatura tem evidenciado correlações negativas entre a complexidade do regime medicamentoso e a adesão ao tratamento. À medida que o número de medicamentos e/ou de doses aumenta, a adesão diminui. A frequência das doses parece ser um fator mais importante para a não adesão do que a quantidade de diferentes fármacos a serem tomados diariamente ${ }^{(4,6)}$.

Estudo transversal, realizado entre 46 adultos brasileiros com DM, em que um dos objetivos foi estimar a prevalência da adesão medicamentosa segundo fatores inerentes à pessoa, ao relacionamento profissional-paciente, ao esquema terapêutico e à doença, por meio de instrumento de autorrelato, mostrou que a prevalência da adesão foi maior $(81,1 \%)$ entre as pessoas que não referiram mudanças na vida diária para o cumprimento do tratamento ${ }^{(5)}$.

Outros fatores que parecem se associar com a baixa adesão ao tratamento medicamentoso do DM são: maior tempo de doença ${ }^{(5,7)}$, o uso de insulina como tratamento medicamentoso $^{(2-3,8)}$ e o mau controle glicêmico ${ }^{(5,9)}$.

Em relação ao tempo de doença, a adesão é menor entre as pessoas com mais de cinco anos de diagnóstico ${ }^{(5,7)}$. A baixa adesão ao uso de insulina se deve à necessidade de mudanças na rotina, especialmente quanto aos horários das refeições ${ }^{(5)}$, à percepção de que a insulinoterapia interfere na realização de atividades sociais e ocupacionais ${ }^{(3)}$, e ao ganho de peso corporal $^{(2)}$. Quanto ao controle glicêmico, estudos mostram que houve maior prevalência de não adesão entre as pessoas com valores de hemoglobina glicada acima de $7 \%{ }^{(5)}$, bem como entre aquelas com a glicemia alterada ${ }^{(9)}$.

\section{OBJETIVO}

O presente estudo teve por objetivo investigar a adesão ao tratamento medicamentoso e sua relação com as variáveis clínicas, de tratamento e o controle metabólico de pessoas com DM2, em seguimento ambulatorial.

\section{MATERIAL E MÉTODOS}

Trata-se de um estudo seccional, de abordagem quantitativa.
A amostra do estudo $(n=162)$ foi por conveniência e selecionada por meio da revisão semanal dos prontuários das pessoas agendadas no Ambulatório de Endocrinologia e Metabologia do Hospital das Clínicas, da Faculdade de Medicina de Ribeirão Preto, da Universidade de São Paulo, no período de maio a novembro de 2008, mediante os critérios de inclusão/ exclusão.

Com o objetivo de homogeneizar a amostra no que se refere à complexidade do tratamento medicamentoso na presença de comorbidades associadas, foram incluídas pessoas com DM2 com ausência de complicações crônicas em estágio avançado, ou seja, tratamento hemodialítico, amaurose, sequelas de Acidente Vascular Cerebral/Insuficiência Cardíaca, amputações prévias ou úlcera ativa em membros inferiores, bem como aquelas em cadeira de rodas ou maca. As pessoas com dificuldade de compreensão dos instrumentos devido a fatores culturais ou incapazes de manter diálogo não foram incluídas no estudo. Optou-se por incluir pessoas com idade mínima de 40 anos pelo fato de o DM2 ser diagnosticado mais frequentemente após essa idade ${ }^{(10)}$.

As pessoas abordadas eram conduzidas a uma sala privativa e, após apresentação dos objetivos do estudo e esclarecimento sobre o anonimato da participação, eram convidadas a participar do estudo. Aos que concordaram, foi entregue o Termo de Consentimento Livre e Esclarecido (TCLE), o qual foi lido em voz alta por um dos pesquisadores, e solicitada a assinatura do mesmo ao final da leitura.

Para a coleta dos dados, utilizou-se o instrumento "Medida de Adesão aos Tratamentos" (MAT), versão traduzida, adaptada e validada $(\alpha=0,74)$ para o português de Portugal ${ }^{(11)}$ e adequada ao português do Brasil ${ }^{(5)}$.

A MAT é uma escala composta por sete itens e se destina a avaliar a adesão ao tratamento medicamentoso prescrito. Todos os itens apresentam um padrão de resposta que vai de "sempre" até "nunca", com uma pontuação variando, respectivamente, de um a seis. A adesão é determinada pela média global do instrumento, ou seja, somam-se os pontos de cada item e divide-se pelo número de itens (sete). Considera-se "adesão" ao tratamento quando a média obtida apresentar valores entre cinco e seis pontos e "não adesão", quando a média for inferior a cinco pontos ${ }^{(11)}$.

As variáveis sociodemográficas (sexo, idade, escolaridade, estado civil, procedência, ocupação e renda familiar mensal) foram coletadas por meio de entrevistas com os participantes, utilizando instrumento estruturado para essa finalidade.

As variáveis antropométricas (índice de massa corporal IMC e circunferência abdominal) foram obtidas por meio de duas aferições, com técnica e equipamento padronizados ${ }^{(12)}$, considerando-se a média das duas aferições. O IMC foi obtido por meio da razão peso/altura ao quadrado, e categorizado em: normal (IMC $<25,0 \mathrm{~kg} / \mathrm{m}^{2}$, incluindo a categoria $>18,5$ $\mathrm{kg} / \mathrm{m}^{2}$ ), sobrepeso (IMC entre 25,0 e $29,9 \mathrm{~kg} / \mathrm{m}^{2}$ ), e obeso $\left(\mathrm{IMC} \geq 30,0 \mathrm{~kg} / \mathrm{m}^{2}\right)^{(12)}$. Para a variável circunferência abdominal, foram considerados adequados os valores de $\leq 102 \mathrm{~cm}$ para homens e $\leq 88 \mathrm{~cm}$ para mulheres, de acordo com o Executive Summary of Third Report of the National Cholesterol Education Program ${ }^{(13)}$. 
As variáveis clínicas foram: tempo de diagnóstico (autodeclarado); presença de complicações/comorbidades, obtida por meio de consulta aos prontuários, e pressão arterial, obtida por meio de duas aferições, com técnica e equipamento padroniza$\operatorname{dos}^{(14)}$, e considerada a média das duas aferições. Para a categorização da pressão arterial, foram utilizadas as recomendações do The Seventh Report of the Joint National Committee on Prevention Detection, Evaluatin and Treatment of High Blood Pressure. $\mathrm{O}$ valor da pressão arterial sistólica considerado normal foi $<130 \mathrm{mmHg}$ e alterado $\geq 130 \mathrm{mmHg}$ e o da pressão arterial diastólica normal foi $<80 \mathrm{mmHg}$ e alterado $\geq 80 \mathrm{mmHg}^{(14)}$.

As variáveis relacionadas ao tratamento foram: tipo de tratamento medicamentoso, tempo de uso de insulina e/ou antidiabéticos orais (ADOs) e frequência diária de administração de insulina e/ou ADOs, todas autodeclaradas pelos participantes.

Como parâmetros de controle metabólico, foram considerados os seguintes dados laboratoriais, obtidos por meio de consulta ao sistema eletrônico interno do local de estudo, categorizados em normal e alterado, mediante os padrões estabelecidos pelos consensos ou diretrizes hemoglobina glicada A1c (HbA1c): método Cromatografia Líquida de Alta Performance (HPLC), valor de referência $<7 \%{ }^{(15)}$; glicemia plasmática de jejum, método enzimático automatizado, valor de referência $<130 \mathrm{mg} / \mathrm{dl}^{(15)}$; colesterol total, método enzimático automatizado, valor de referência $<200 \mathrm{mg} / \mathrm{dl}^{(10)}$; colesterol de alta densidade (HDL), método enzimático automatizado, valor de referência $>45$ $\mathrm{mg} / \mathrm{dl}^{(10)}$; colesterol de baixa densidade (LDL), método enzimático automatizado, valor de referência $<100 \mathrm{mg} / \mathrm{dl}^{(10,15)}$; triglicerídeos, método enzimático automatizado, valor de referência $<150 \mathrm{mg} / \mathrm{dl}^{(10)}$.

Os dados coletados foram armazenados no programa Excel, com dupla digitação e validação dos mesmos. Posteriormente, o banco de dados foi exportado para o programa Statistical Package for Social Science (SPSS), versão 11.5, para análise exploratória uni e bivariada com frequências, medidas de tendência central (média e mediana) e de variabilidade (desvio-padrão). Para verificar a existência de relação dos escores da MAT com as variáveis clínicas, de tratamento de controle metabólico, utilizaram-se testes de correlação linear. A força das correlações foi verificada de acordo com a seguinte classificação: fraca $(r<0,3)$, moderada $(0,3<r<0,6)$ e forte $(r>0,6)^{(16)}$. O nível de significância adotado foi de 0,05.

O estudo foi aprovado pelo Comitê de Ética do Hospital das Clínicas da Faculdade de Medicina de Ribeirão Preto da Universidade de São Paulo (No 2049/2008).

\section{RESULTADOS}

\section{Caracterização sociodemográfica, clínica, de tratamento} e controle metabólico

Em relação à caracterização sociodemográfica, destaca-se que a amostra estudada ficou constituída por 94 (58\%) pessoas do sexo feminino e $68(42 \%)$ do sexo masculino; 114 (70,4\%) eram casados; 129 (79,6\%) procedentes de Ribeirão Preto e região, com média de idade de $59,4\left(\mathrm{DP}^{*}=8,02\right)$ anos e de escolaridade de 5,4 (DP=3,9) anos. A renda mensal familiar média referida foi de $\mathrm{R} \$ 1.325,65$ ( $\mathrm{DP}=1.122,72$ ); quanto à situação de trabalho, $67(41,4 \%)$ eram aposentados ou pensionistas, 55 (34\%) trabalhavam em casa sem remuneração, e 31 (19,1\%) eram ativos.

As variáveis clínicas, de tratamento e controle metabólico estão apresentadas nas Tabelas 1, 2 e 3, respectivamente.

Tabela 1 - Caracterização da amostra estudada segundo as variáveis clínicas, Ribeirão Preto-SP, 2008

\begin{tabular}{lc}
\hline Variáveis (n= 162) & Média (DP*) ou Número (\%) \\
\hline IMC $\left(\mathrm{kg} / \mathrm{m}^{2}\right)$ & $31,74(\mathrm{DP}=6,2)$ \\
Pressão arterial sistólica $(\mathrm{mmHg})$ & $140,34(\mathrm{DP}=22,4)$ \\
Pressão arterial diastólica $(\mathrm{mmHg})$ & $77,64(\mathrm{DP}=11,8)$ \\
Circunferência abdominal $(\mathrm{cm})$ & $106,4(\mathrm{DP}=12,9)$ \\
Tempo de diagnóstico (em anos) & $14,8(\mathrm{DP}=7,8)$ \\
Complicações / comorbidades ${ }^{\dagger}$ & \\
Hipertensão arterial & $142(87,7 \%)$ \\
Dislipidemias & $118(72,8 \%)$ \\
Cardiovasculares & $85(52,5 \%)$ \\
Renais & $83(51,2 \%)$ \\
Obesidade & $75(46,3 \%)$ \\
Oftálmicas & $66(40,7 \%)$ \\
Neurológicas & $43(26,5 \%)$ \\
Pé diabético & $9(5,6 \%)$ \\
Outras doenças & $98(60,5 \%)$ \\
\hline
\end{tabular}

* DP: desvio-padrão.

† As categorias não são mutuamente excludentes.

Em relação ao IMC, quando categorizado em "normal", "sobrepeso" e "obeso", observou-se que aproximadamente 88 $(54,3 \%)$ e $58(35,8 \%)$ participantes encontravam-se, respectivamente, nas categorias de obeso e sobrepeso. A pressão arterial sistólica foi considerada normal para $58(35,8 \%)$ participantes, e a diastólica, para $100(61,7 \%)$ pessoas. Referente à circunferência abdominal, os valores normais para homens e mulheres foram encontrados, respectivamente, em 24 (35,3\%) e sete $(7,4 \%)$ participantes. O tempo de diagnóstico variou entre um e 40 anos, com média de 14,8 anos. Entre as complicações/ comorbidades, as de maiores frequências foram a hipertensão arterial sistêmica $(87,7 \%)$ e as dislipidemias (72,8\%) (Tabela 1). Salienta-se que a categoria "outras doenças" compreende aquelas não relacionadas ao DM como, por exemplo, gastrite/úlcera péptica, cirrose hepática, artrite reumatóide, entre outras.

A associação de insulina e ADOs foi o tratamento medicamentoso de maior frequência $(64,2 \%$ dos participantes). Em relação aos ADOs, a classe terapêutica mais utilizada, independente de associações, foi a das Biguanidas (62,3\%). Quanto à insulina, o tipo utilizado pela maioria dos participantes (58\%) foi a NPH/Lenta (Tabela 2 ).

Para os dados laboratoriais analisados, com exceção do colesterol total e do LDL colesterol, a média dos resultados se encontrou acima dos valores de normalidade para a maioria 
Tabela 2 - Caracterização da amostra estudada segundo as variáveis de tratamento, Ribeirão Preto-SP, 2008

\begin{tabular}{lc}
\hline \multicolumn{1}{c}{ Variáveis (n= 162) } & $\begin{array}{c}\text { Média (DP*) ou Número } \\
(\%)\end{array}$ \\
\hline Tipo de tratamento medicamentoso & $24(14,8 \%)$ \\
ente antidiabético oral (ADO) & $34(21,0 \%)$ \\
Somente insulina & $104(64,2 \%)$ \\
Associações (insulina + ADO) & \\
Uso de insulina & $87,2(\mathrm{DP}=83,5)$ \\
Tempo de uso (em meses) & $1,9(\mathrm{DP}=0,4)$ \\
Frequência diária de administração & \\
Tipo de insulina & $94(58 \%)$ \\
NPH/Lenta & $42(25,9 \%)$ \\
NPH + Regular & $1(0,6 \%)$ \\
Lantus/Ultralenta & $1(0,6 \%)$ \\
Não soube informar & \\
Uso de ADO & $87(\mathrm{DP}=65,8)$ \\
Tempo de uso (em meses) & $2,6(\mathrm{DP}=0,7)$ \\
Frequência diária de administração & \\
Tipo de ADO & $101(62,3 \%)$ \\
Biguanidas & $26(16 \%)$ \\
Sulfoniluréias & $1(0,6 \%)$ \\
Toglitazonas &
\end{tabular}

* DP: desvio-padrão.

Tabela 3 - Caracterização da amostra estudada segundo os parâmetros de controle metabólico, Ribeirão PretoSP, 2008

\begin{tabular}{lc}
\hline Variáveis $(\mathbf{n}=\mathbf{1 6 2})$ & Média $(\mathbf{D P} *)$ ou Número $(\%)$ \\
\hline Hemoglobina glicada $(\%)$ & $9,1(\mathrm{DP}=1,8)$ \\
Glicemia plasmática de jejum $(\mathrm{mg} / \mathrm{dl})$ & $178(\mathrm{DP}=57,9)$ \\
Colesterol total $(\mathrm{mg} / \mathrm{dl})$ & $182(\mathrm{DP}=47,7)$ \\
HDL Colesterol $(\mathrm{mg} / \mathrm{dl})$ & $42(\mathrm{DP}=9,3)$ \\
LDL Colesterol $(\mathrm{mg} / \mathrm{dl})$ & $100(\mathrm{DP}=32,1)$ \\
Triglicérides $(\mathrm{mg} / \mathrm{dl})$ & $215(\mathrm{DP}=237,3)$ \\
\hline
\end{tabular}

* DP: desvio-padrão.

dos participantes, indicando um controle metabólico inadequado. Os valores normais de hemoglobina glicada e de glicemia plasmática de jejum foram observados, respectivamente, em $17(10,5 \%)$ e 34 (21\%) participantes.

\section{Adesão ao tratamento medicamentoso}

Em um intervalo possível de 1 a 6, obteve-se o de 3,29 a $6,0$, com média de 5,66 ( $\mathrm{DP}=0,42)$, sugerindo alta adesão ao tratamento medicamentoso. Dessa forma, observou-se que $155(95,7 \%)$ participantes foram classificados na categoria “adesão" e 7 (4,3\%), na "não adesão".

Relação das variáveis clínicas, de tratamento e controle metabólico com a adesão ao tratamento medicamentoso

A Tabela 4 fornece os valores das correlações (r) das variáveis clínicas, de tratamento e controle metabólico, de natureza numérica, com a adesão ao tratamento medicamentoso (MAT), a um nível de significância de 0,05.

Tabela 4 - Correlação entre a MAT e as variáveis clínicas, de tratamento e controle metabólico na amostra estudada, Ribeirão Preto-SP, 2008

\begin{tabular}{lrl}
\hline \multicolumn{1}{c}{ MAT (N=162) } & $\mathbf{( r )}^{\text {中 }}$ & $\boldsymbol{p}$-valor \\
\hline IMC & $-0,04$ & 0,61 \\
Pressão arterial sistólica & $-0,06$ & 0,40 \\
Pressão arterial diastólica & $-0,15$ & $0,04 *$ \\
Circunferência abdominal & $-0,06$ & 0,46 \\
Tempo de diagnóstico & 0,08 & 0,32 \\
Tempo de uso de insulina & 0,04 & 0,67 \\
Frequência diária de administração de insulina & 0,18 & $0,03 *$ \\
Tempo de uso de antidiabético oral & $-0,04$ & 0,69 \\
Frequência diária de administração dos antidiabéticos orais & 0,27 & $0,00 *$ \\
Hemoglobina glicada & $-0,03$ & 0,65 \\
Glicemia plasmática de jejum & $-0,03$ & 0,66 \\
Colesterol total & $-0,01$ & 0,91 \\
HDL Colesterol & $-0,04$ & 0,59 \\
LDL Colesterol & $-0,07$ & 0,36 \\
Triglicérides & $-0,03$ & 0,73 \\
\hline
\end{tabular}

: Corresponde aos coeficientes de correlação de Pearson ou Spearman, conforme classificação da amostra estudada.

*: Significância estatística.

Para um $\mathrm{p}<0,05$, foram obtidas correlações diretas e estatisticamente significantes entre adesão ao tratamento medicamentoso e frequência diária de administração de insulina, bem como entre adesão e frequência diária de administração do antidiabético oral, sendo ambas de fraca magnitude. De modo similar, observou-se correlação inversa e de fraca magnitude entre adesão ao tratamento e pressão arterial diastólica, sugerindo que quanto maior o escore de adesão, menor o valor dessa última variável. Não foram observadas diferenças estatisticamente significantes entre os distintos tipos de tratamento medicamentoso (antidiabéticos orais, insulina e associações), no que se refere aos escores de adesão ao tratamento.

\section{DISCUSSÃO}

Na amostra estudada, a média obtida para a adesão ao tratamento medicamentoso foi de 5,66 ( $\mathrm{DP}=0,42)$, em uma escala pontuada de 1 a 6 , na qual escores mais altos indicam maior adesão. Dos 162 participantes, 155 (95,7\%) foram considerados em "adesão" e como a média está próxima do valor máximo possível, pode-se dizer que a amostra estudada possui uma alta adesão medicamentosa.

Estudos nacionais, desenvolvidos em amostras populacionais com DM e que utilizaram métodos similares aos do presente estudo, encontraram, respectivamente, $78,3 \%$ e $84,4 \%$ de participantes em adesão ${ }^{(5,17)}$. A alta adesão medicamentosa pode ser atribuída aos modelos mentais (percepções e crenças) das pessoas com DM sobre o seu tratamento, em que as pessoas consideram a medicação como a modalidade de 
tratamento de maior impacto no controle do DM, ao comparar com a dieta e o exercício físico ${ }^{(18)}$.

No que se refere à relação entre a adesão e as variáveis clínicas, não foram observadas correlações entre adesão medicamentosa e tempo de diagnóstico no presente estudo, para um valor de $\mathrm{p}<0,05$, diferentemente do estudo transversal anteriormente citado $^{(5)}$ e do estudo que avaliou os efeitos de uma intervenção educativa na adesão ao tratamento de imigrantes turcos com DM2 ${ }^{(7)}$, nos quais foi identificado que os participantes com mais de cinco anos de diagnóstico apresentavam menor adesão. Entretanto, outro estudo nacional ${ }^{(17)}$, que objetivou investigar a associação entre adesão ao tratamento de pessoas com DM2 e variáveis sociodemográficas, clínicas e de controle metabólico, não encontrou associação entre a adesão e o tempo de diagnóstico. O estudo seccional(19), desenvolvido entre adultos portugueses com DM, cujo objetivo foi avaliar a relação de características demográficas e clínicas com a adesão ao tratamento, por meio de um instrumento de autorrelato, também não identificou correlações entre a adesão e o tempo de doença. Estes resultados dos dois últimos estudos ${ }^{(17,19)}$, portanto, se assemelham aos da presente investigação.

De modo similar, não foram observadas correlações estatisticamente significantes entre a adesão e a presença de complicações crônicas do DM no grupo estudado, divergindo do estudo realizado em Portugal(19), no qual a adesão medicamentosa foi maior entre os participantes que possuíam complicações crônicas da doença $(p<0,05)$.

Observou-se correlação inversa e estatisticamente significante, porém de fraca magnitude, entre a adesão ao tratamento e os valores médios de pressão arterial diastólica, sugerindo que, quanto maior a adesão, menor a medida da pressão. Esse achado corrobora pesquisa que teve por objetivo determinar a adesão medicamentosa de adultos norte-americanos com DM e sua relação com a quantidade de fármacos prescritos e controle metabólico ${ }^{(20)}$, a qual também identificou menor medida de pressão arterial diastólica entre os participantes com maior adesão medicamentosa no DM, embora tenha utilizado outro instrumento para avaliar a adesão. Resultados divergentes foram encontrados em estudo nacional(17) que utilizou o mesmo instrumento da presente investigação para avaliar a adesão medicamentosa, e que não constatou associação entre esta variável e a pressão arterial.

Referente às variáveis de tratamento, não se observaram diferenças nos escores de adesão entre os distintos tipos de tratamento medicamentoso (somente insulina, somente antidiabético oral e associações), para um valor de $p<0,05$. Esse resultado é discrepante de um estudo de revisão sistemática sobre adesão medicamentosa no $\mathrm{DM}^{(2)}$, que encontrou um menor percentual de adesão entre pessoas em insulinoterapia do que aquelas em uso de antidiabéticos orais (73\% e $86 \%$, respectivamente). Outra investigação ${ }^{(8)}$ também encontrou menor percentual de adesão ao uso de insulina (54,7\% para DM1 e 36,1\% para DM2) do que ao uso de antidiabéticos orais (84,1\%, somente para DM2), ou à terapia combinada (32,5\% para DM1 e 45,9\% para DM2), ao avaliar a adesão medicamentosa de adultos portugueses com DM. No entanto, as autoras não mencionam se as diferenças foram estatisticamente significantes.
Foram verificadas correlações diretas e estatisticamente significantes, porém de fraca magnitude, entre adesão e frequência diária de aplicação de insulina, bem como entre adesão e frequência diária de tomada do ADO. Esses resultados parecem indicar que quanto maior a frequência das doses, maior a adesão, divergindo da literatura ${ }^{(2,4,6)}$. No estudo que objetivou estimar a adesão medicamentosa de adultos norte-americanos com DM2 e sua relação com a quantidade de fármacos prescritos ${ }^{(20)}$, não houve associação entre a quantidade de medicamentos e a taxa de adesão. Ao contrário da presente investigação, estudos relacionados à adesão terapêutica entre pessoas com DM mostram uma correlação negativa entre a complexidade do regime medicamentoso e a adesão das pessoas, ou seja, à medida que o número de medicamentos e/ou de doses aumenta, a adesão diminui ${ }^{(4,6,21)}$.

Contudo, dois estudos de revisão ${ }^{(4,22)}$ sobre a adesão medicamentosa no DM, sendo um de revisão sistemática da literatura ${ }^{(22)}$, apontam que, apesar de uma única dosagem diária favorecer a adesão ${ }^{(4,22)}$, o risco de super dosagem torna-se maior devido ao esquecimento ${ }^{(22)}$. Para alguns autores ${ }^{(23-24)}$, a super dosagem é considerada um tipo de não adesão.

Em relação às variáveis de controle metabólico, não foram observadas correlações com a adesão ao tratamento, corroborando outras investigações ${ }^{(7,17,20)}$. Em contrapartida, outros dois estudos nacionais e transversais ${ }^{(5,9)}$ mostraram maior prevalência de não adesão entre as pessoas com valores de hemoglobina glicada acima de $7 \%$, ainda que sem significância estatística, bem como entre aquelas com a glicemia alterada $(p=0,013)^{(9)}$.

Estudo seccional ${ }^{(21)}$ realizado entre 11.896 adultos franceses com DM2 em seguimento ambulatorial, que objetivou avaliar a adesão aos antidiabéticos orais, por meio de instrumento de autorrelato, e sua relação com o controle metabólico, também encontrou associação entre a adesão e os valores de hemoglobina glicada. No estudo referido ${ }^{(21)}$, quanto maior a adesão, melhor o controle metabólico $(p<0,01)$.

Outro estudo seccional ${ }^{(6)}$, em que um dos objetivos foi analisar a relação da adesão medicamentosa com o controle glicêmico de adultos escoceses com DM2, mostrou que uma melhor adesão ao uso de insulina estava relacionada a menores valores de hemoglobina glicada $(p<0,0001)$.

Mediante as divergências encontradas entre os estudos, é importante considerar que tanto a adesão ao tratamento, quanto o controle metabólico são fenômenos complexos, os quais podem ser influenciados por fatores socioculturais, ambientais e psicológicos, tais como as crenças em saúde ${ }^{(25)}$. Salienta-se, também, que a ausência de uma definição consensual de adesão, a variedade de métodos de avaliação e a escassez de estudos utilizando o mesmo instrumento, especialmente em amostras brasileiras de pessoas com DM, dificultaram a ampliação da discussão dos dados do presente estudo.

\section{CONCLUSÃO}

A ausência de correlações entre adesão e algumas características clínicas, especialmente o tempo de diagnóstico, é um achado divergente da literatura, uma vez que esta última variável é apontada como um fator dificultador da adesão, quando de longa data. Estudos também têm mostrado correlação 
inversa entre a adesão e a complexidade do regime medicamentoso, ao contrário do presente estudo. De modo similar, ao analisar a relação da adesão ao tratamento com o controle metabólico, não foram observadas correlações significativas para um valor de $p<0,05$. A literatura aponta resultados controversos a respeito dessa relação, ora se assemelhando ao presente estudo, ora divergindo do mesmo.

Acredita-se que, além da adesão ao tratamento, outras variáveis possam influenciar o controle metabólico. Outra questão relevante é que a adesão ao tratamento, ao contrário do controle metabólico, é vista como um comportamento humano e, como tal, está sujeito às influências do ambiente social em que vive a pessoa. Nessa perspectiva, o estabelecimento de uma relação terapêutica que permita conhecer a pessoa em seu contexto de vida diária, é fundamental para se promover a adesão ao tratamento. $\mathrm{O}(\mathrm{a})$ enfermeiro(a), pela natureza de seu trabalho, constitui o elo de ligação entre a pessoa com doença crônica, sua família e os serviços de saúde, tendo o desafio de auxiliar a pessoa a modificar seu estilo de vida e manejar sua doença.

Diante do exposto, a adesão ao tratamento é um tema que merece mais investigações. Dessa forma, pesquisas na área devem ser incentivadas, com amostras populacionais maiores, a fim de ampliar as análises das relações entre adesão ao tratamento e as demais variáveis, bem como estudar as influências das intervenções de enfermagem no comportamento de adesão, necessário para a melhoria do estado de saúde.

\section{REFERÊNCIAS}

1. Haynes RB. Introduction: the definition of compliance. In: Haynes RB, Taylor DW, Sackett DL. Compliance in Health Care. Baltimore (US): The Johns Hopkins University Press; 1979. p.1-6.

2. Cramer JA. A Systematic review of adherence with medications for diabetes. Diabetes Care. 2004; 27(5):1218-24.

3. Peyrot M, Rubin RR, Kruger DF, Travis LB. Correlates of insulin injection omission. Diabetes Care. 2010;33(2):240-5.

4. Rubin RR. Adherence to pharmacologic therapy in patients with type 2 diabetes mellitus. Am J Med. 2005;118 Suppl 5A:27S-34S.

5. Gimenes HT, Zanetti ML, Haas VJ. Factors related to patient adherence to antidiabetic drug therapy. Rev Latinoam Enferm. 2009;17(1):46-51.

6. Donnelly LA, Morris AD, Evans JMM. Adherence to insulin and its association with glycaemic control in patients with type 2 diabetes. QJM. 2007;100(6):345-50.

7. Uitewaal P, Hoes A, Thomas S. Diabetes education on Turkish immigrant diabetics: predictors of compliance. Patient Educ Couns. 2005;57(2):158-61.

8. Ó DN, Loureiro I. Adesão ao regime terapêutico da diabetes. Rev Port Diabetes. 2007;2(2):18-21.

9. Araújo MFM, Freitas RWJF, Fragoso LVC, Araújo TM, Damasceno MMC, Zanetti ML. Cumprimento da terapia com antidiabéticos orais em usuário da atenção primária. Texto \& Contexto Enferm. 2011; 20(1):135-43.

10. Sociedade Brasileira de Diabetes. Diretrizes da Sociedade Brasileira de Diabetes. São Paulo: AC Farmacêutica; 2013. 385 p.

11. Delgado $A B$, Lima ML. Contributo para a validação concorrente de uma medida de adesão aos tratamentos. Psic Saúde \& Doenças. 2001;2(2):81-100.

12. World Health Organization. Physical status: the use and interpretation of anthropometry. Geneva: WHO; 1995.

13. Expert Panel on Detection, Evaluation and Treatment of High Blood Cholesterol in Adults. Executive Summary of the Third Report of the National Cholesterol Education Program (NCEP) Expert Panel on Detection, Evaluation, and Treatment of High Blood Cholesterol in Adults (Adult Treatment Panel III). JAMA. 2001;285(19):2486-97.

14. Chobanian AV, Bakris GL, Black HR, Cushman WC, Green LA, Izzo Jr JL, et al. The Seventh Report of Joint National Committee on Prevention, Detection, Evaluation and Treatment of High Blood Pressure. JAMA. 2003;289(19):2560-72.

15. American Diabetes Association. Standards of Medical Care in Diabetes - 2014. Diabetes Care. 2014;37 Suppl $1: S 14-S 80$

16. Zou KH, Tuncali K, Silverman SG. Correlation and Simple Linear Regression. Radiology. 2003; 227(3):617-22.

17. Faria HTG, Rodrigues FFL, Zanetti ML, Araujo MFM, Damasceno MMC. Factors associated with adherence to treatment of patients with diabetes mellitus. Acta Paul Enferm. 2013;26(3):231-7.

18. Bradbent E, Donkin L, Stroh JC. Illness and treatment perceptions are associated with adherence to medications, diet, and exercise in diabetic patients. Diabetes Care. 2011;34(2):338-40.

19. Silva I, Pais-Ribeiro J, Cardoso H. Adesão ao tratamento do diabetes mellitus: a importância das características demográficas e clínicas. Referência. 2006;2(2):34-41.

20. Grant RW, Devita NG, Singer DE, Meigs JB. Polypharmacy and medication adherence in patients with type 2 diabetes. Diabetes Care. 2003;26(5):1408-12.

21. Guillausseau PJ. Influence of oral antidiabetic drugs compliance on metabolic control in type 2 diabetes: a survey in general practice. Diabetes Metab. 2003;29(1):79-81.

22. Odegard PS, Capoccia K. Medication taking and diabetes: a systematic review of the literature. Diabetes Educ. 2007;33(6):1014-29.

23. Clark M. Adherence to treatment in patients with type 2 diabetes. J Diabetes Nurs. 2004;8(10):135-40.

24. Moosa MYH, Jeenah FY, Kazadi N. Treatment adherence. SAJP. 2007;13(2):40-5.

25. Gomes-Villas Boas LC, Foss MC, Freitas MC, Pace AE. Relationship among social support, treatment adherence and metabolic control of diabetes mellitus patients. Rev Latinoam Enferm. 2012;20(1):52-8. 


\section{ERRATA}

No volume 67, n 2, pp. 268-273, artigo Boas LCG-V, Foss-Freitas MC, Pace AE. Adesão de pessoas com diabetes mellitus tipo 2 ao tratamento medicamentoso, onde se lê, à página 270, “... utilizaram-se testes de correlação linear e de comparação das médias entre os grupos (retirar esse trecho, pois não foi utilizado)", leia-se:

... utilizaram-se testes de correlação linear. 